\title{
Poor Sucking Reflex
}

National Cancer Institute

\section{Source}

National Cancer Institute. Poor Sucking Reflex. NCI Thesaurus. Code C117310.

Uncoordinated or ineffective drawing of saliva or breast milk by a newborn infant. 